\title{
Hemodiluição normovolêmica aguda em cirurgias de deformidade da coluna*
}

\section{Acute Normovolemic Hemodilution in Spinal Deformity Surgery}

\author{
Marianna Fergutz Santos Batista ${ }^{1}{ }^{\circledR}$ Caroline Oliveira Costa ${ }^{1} \quad$ Emiliano Neves Vialle ${ }^{1}$ \\ Joana Bretas Rondon Cabral Guasque ${ }^{1}$ Joana Zulian Fiorentin ${ }^{1}$ Camila de Santiago Souza ${ }^{1}$ \\ ${ }^{1}$ Serviço de Ortopedia e Traumatologia, Hospital Universitário Cajuru, \\ Pontifícia Universidade Católica do Paraná (PUC-PR), Curitiba, PR, \\ Endereço para correspondência Marianna Fergutz dos Santos \\ Batista, MD, Serviço de Ortopedia e Traumatologia, Hospital \\ Universitário Cajuru, Pontifícia Universidade Católica do Paraná \\ Brasil \\ (PUC-PR), Curitiba, PR, Brasil (e-mail: mari_fbatista@hotmail.com).
}

Rev Bras Ortop 2019;54:516-523.

\section{Resumo \\ Palavras-chave \\ - hemodiluição/ métodos \\ - sangramento \\ - transfusão de sangue autóloga \\ - coluna vertebral \\ - escoliose}

\section{Abstract}

Objetivo Comparar de modo prospectivo os parâmetros clínicos e laboratoriais dos pacientes submetidos a hemodiluição normovolêmica aguda associada ao ácido tranexâmico com um grupo de controle que recebeu apenas ácido tranexâmico, durante cirurgia de correção de deformidades da coluna, e avaliar a influência da técnica de hemodiluição no sangramento perioperatório e a necessidade de transfusão de sangue homólogo.

Materiais e Métodos Estudo prospectivo comparativo, com pacientes entre 12 e 65 anos submetidos a cirurgia para correção de deformidades da coluna vertebral, com a técnica de hemodiluição normovolêmica aguda associada ao ácido tranexâmico, versus grupo de controle com ácido tranexâmico isolado na dose de $15 \mathrm{mg} / \mathrm{kg}$. Exames laboratoriais foram feitos e analisados em três momentos de avaliação diferentes.

Resultados Participaram deste estudo 30 pacientes: 17 no grupo de hemodiluição e 13 no grupo de controle. O tempo médio de cirurgia foi maior para o grupo de hemodiluição. O número de níveis operados variou entre 7 e 16 no grupo de hemodiluição, e entre 4 e 13 no grupo de controle. Fez-se osteotomia, predominantemente posterior, em 20 pacientes. O valor médio de sangramento intraoperatório foi maior no grupo de controle. Os parâmetros clínicos se mantiveram estáveis durante todos os procedimentos. Apenas 6 pacientes necessitaram de transfusão sanguínea homóloga, a maioria dos quais pertencia ao grupo de controle $(p>0,05)$.

Conclusão Não houve diferença significativa entre os dois grupos quanto à necessidade de transfusão e sangramento intraoperatório. A gravidade da deformidade foi o principal fator determinante da transfusão.

Objective To prospectively compare the clinical and laboratorial aspects of patients undergoing spine deformity surgery, using the acute normovolemic hemodilution technique with tranexamic acid, versus a control group with tranexamic acid alone, and to evaluate the influence of hemodilution in intraoperative bleeding and the need for homologous transfusion.

Publicado Originalmente por Elsevier Editora Ltda.

recebido

10 de Dezembro de 2017 aceito

21 de Fevereiro de 2018
DOI https://doi.org/

10.1016/j.rbo.2018.02.004. ISSN 0102-3616.
Copyright $\odot 2019$ by Sociedade Brasileira License terms de Ortopedia e Traumatologia. Published by Thieme Revnter Publicações Ltda, Rio de Janeiro, Brazil 


\section{Keywords \\ - hemodilution/ methods \\ - bleeding \\ - autologous blood transfusion \\ - spine \\ - scoliosis}

Materials and Methods Comparative prospective study with patients aged between 12 and 65 years undergoing spine deformity surgery with the acute normovolemic hemodilution technique associated with tranexamic acid versus a control group to which only tranexamic acid $(15 \mathrm{mg} / \mathrm{kg})$ was administered. Laboratorial exams were performed and analyzed in three different moments.

Results A total of 30 patients were included in the present study: 17 in the hemodilution group, and 13 in the control group. The mean duration of the surgery in the hemodilution group was longer. The number of levels submitted to surgery ranged from 7 to 16 in the hemodilution group, and from 4 to 13 in the control group. Osteotomy, predominantly posterior, was performed in 20 patients. There was more intraoperative bleeding in the control group. All patients were stable during the procedures. Only 6 participants needed homologous blood transfusion, mostly from the control group $(p>0.05)$.

Conclusion There was no significant difference between the two groups regarding the need for blood transfusion and intraoperative bleeding. The severity of the deformity was the main determinant for homologous blood transfusion.

\section{Introdução}

Cirurgias de grande porte, como as correções de deformidades da coluna, podem levar a um aumento do sangramento. Para controle da hemostasia, podem ser usados agentes antifibrinolíticos, e também reposição volêmica endovenosa com soluções acelulares ou com hemoderivados homólogos ou autólogos. ${ }^{1}$

A transfusão homóloga expõe o paciente ao risco de reações pulmonares, alérgicas, hemolíticas, imunoalérgicas e aquisição de doenças infectocontagiosas. ${ }^{2}$ A transfusão autóloga pode ser obtida com baixo custo operacional por doações prévias, reaproveitamento do sangue perioperatório, ou hemodiluição normovolêmica aguda (HNA), com a qual se evitam complicações. ${ }^{3}$

A técnica de HNA consiste na retirada de sangue imediatamente antes ou após a indução anestésica, ${ }^{3}$ seguida da sua diluição com coloides e/ou cristaloides, sem reduzir o volume circulante. ${ }^{4}$ Há indicação de seu uso para cirurgias com risco aumentado de sangramento. ${ }^{5}$

O objetivo desde trabalho é comparar de modo prospectivo os parâmetros clínicos e laboratoriais de pacientes submetidos a HNA associada ao ácido tranexâmico com grupo de controle com o uso de ácido tranexâmico isolado, durante cirurgia corretiva de deformidades da coluna. São objetivos também avaliar a influência da técnica no sangramento e a necessidade de transfusão homóloga, e identificar reações adversas e complicações.

\section{Materiais e Métodos}

O presente estudo foi aprovado pelo Comitê de Ética da nossa instituição por meio da Plataforma Brasil, com o número do Certificado de Apresentação para Apreciação Ética (CAAE47883615.0.0000.0020).

Foram incluídos 30 pacientes submetidos a cirurgia eletiva para corrigir deformidades na coluna; eles tinham idade entre
12 e 65 anos, e foram divididos, segundo a classificação da American Society of Anestesiology (ASA), como ASA I, II ou III, sem contraindicações para a técnica anestésica/cirúrgica proposta. Foram critérios de exclusão: risco aumentado de doença arterial coronariana, cerebrovascular e valvulopatia, e portadores de insuficiência renal aguda, broncopneumonia e coagulopatias.

Os pacientes foram divididos aleatoriamente entre os grupos de HNA e de controle. Todos foram submetidos a anestesia geral venosa total com remifentanil $(0,1-0,3 \mathrm{mcg} /$ $\mathrm{kg} / \mathrm{min}$ ), propofol (100-200 mcg/ $/ \mathrm{kg} / \mathrm{min}$ ) e cisatracúrio (ataque: $0,15 \mathrm{mg} / \mathrm{kg}$; repique: $0,03 \mathrm{mg} / \mathrm{kg}$ ) quando o potencial evocado não foi monitorado. Após a indução anestésica, todos receberam $15 \mathrm{mg} / \mathrm{kg}$ de ácido tranexâmico e $0,1 \mathrm{mg} /$ $\mathrm{kg}$ de morfina. $\mathrm{O}$ uso de medicamentos adjuvantes, por não interferir nos resultados, ficou a critério do anestesiologista. Foram monitorados a frequência cardíaca, o bloqueio neuromuscular (train of four), a oximetria de pulso, a cardioscopia, a variação do segmento ST, além da pressão arterial invasiva e da diurese por sonda.

Foram feitos exames laboratoriais (hemoglobina [Hb], hematócrito $[\mathrm{Ht}]$, tempo de atividade da protrombina [TAP], tempo de tromboplastina parcial ativada [TTPA], sódio, potássio, magnésio, cálcio iônico, gasometria arterial e lactato) logo após a indução anestésica, após a coleta de sangue da HNA, no pós-operatório imediato, e 24 horas após.

A HNA foi feita em 17 pacientes, baseada na fórmula de Gross (-Fig. 1), com coleta entre $80 \%$ e $100 \%$ do volume a ser

$$
V=V S E \times \frac{H \text { to }-H t f}{H t m}
$$

Fig. 1 Fórmula de Gross. Abreviaturas: V, volume de sangue a ser retirado; VSE, volume de sangue estimado $(65 \mathrm{~mL} / \mathrm{kg}$ em mulheres, e $70 \mathrm{~mL} / \mathrm{kg}$ em homens); Hto, hematócrito inicial; Htf, hematócrito final; Htm, hematócrito médio (diferença entre Hto e Htf). 
retirado via artéria ou veia periféricas (máximo de $500 \mathrm{~mL}$ por bolsa coletada). ${ }^{4,5}$ Em caso de instabilidade hemodinâmica, a HNA seria suspensa. As bolsas foram identificadas conforme ordem de coleta, estocadas em caixa térmica, e reinfundidas na ordem inversa à da coleta. Fez-se a hemodiluição na proporção de 3:1 com solução de soro fisiológico 0,9\% e Ringer lactato.

A reposição volêmica adicional foi calculada para cobrir o jejum pré-operatório, o porte cirúrgico $(6 \mathrm{~mL} / \mathrm{kg} / \mathrm{h}),{ }^{4,5}$ enquanto que a perda volêmica no perioperatório (proporção de 3:1), foi reposta apenas se débito urinário estivesse $<0,5-1,0 \mathrm{~mL} / \mathrm{kg} / \mathrm{he}$ e/ ou no caso de instabilidade hemodinâmica.

Quanto à técnica cirúrgica, a decisão entre instrumentação pedicular ou associação com osteotomias (de elementos posteriores, subtração pedicular ou ressecção do corpo vertebral [RCV]) foi determinada conforme a deformidade.

A transfusão de sangue homólogo foi feita apenas se a concentração de $\mathrm{Hb}$ fosse $<7 \mathrm{mg} / \mathrm{dL}$ (ou $9 \mathrm{mg} / \mathrm{dL}$ em caso de idosos ou de baixa reserva cardiovascular), ou se houvesse alterações hemodinâmicas ou persistência de sangramento.

Os dados foram tabulados no programa Microsoft Excel 2016 (Microsoft Corp., Redmond WA, EUA). Para a comparação das variáveis quantitativas, foi usado o teste $t$ de Student ou o teste não paramétrico de Mann-Whitney. Para as variáveis categóricas, usou-se o teste exato de Fisher. A condição de normalidade das variáveis foi avaliada pelo teste de Kolmogorov-Smirnov. Valores de $p<0,05$ indicaram significância estatística. Os dados foram analisados com o programa Statistical Package for the Social Sciences (SPSS, IBM Corp., Armonk, NY, EUA), versão 20. As variáveis quantitativas foram descritas por médias, medianas, mínimos, máximos e desvios padrão. As variáveis qualitativas foram descritas por frequências e percentuais. Após essa análise, foi feita a comparação com dados da literatura.

\section{Resultados}

A amostra foi composta por 30 pacientes com idades entre $12 \mathrm{e}$ 61 anos (média de 27,1 para o grupo de HNA, e de 21,2 para o grupo de controle; $p>0,05)$, predominantemente do sexo feminino (76,6\%). As principais etiologias foram escoliose idiopática do adolescente (EIA) e escoliose congênita(EC), conforme mostra a - Figura 2. A maioria dos pacientes negou comorbidades (76,6\%). Foram retirados, em média, 622,6 mL de sangue para a hemodiluição (400-940 mL). Os parâmetros clínicos se mantiveram estáveis durante todos os procedimentos. 0 tempo médio de cirurgia para o grupo de HNA foi de $5,2 \mathrm{~h}$, enquanto para o grupo de controle foi de $4,4 \mathrm{~h}(p>0,05)$. O número de níveis operados variou entre 7 e 16 (média: 10,7) para o grupo de HNA, e entre 4 e 13 para o grupo de controle (média: 9,58). Quanto ao número de níveis instrumentados, ele variou entre 6 e 14 (média: 9,82) para o grupo de HNA, e entre 4 e 13 para o grupo de controle (média: 9,66).

Fez-se osteotomia em 20 pacientes, conforme descrito na - Tabela 1. Para fins de análise, graduou-se cada tipo de osteotomia pela sua magnitude: 0 para nenhuma; 1 ponto para cada osteotomia posterior; 2 pontos para subtração pedicular; e 3 pontos para cada corpo vertebral ressecado; assim, esses pacientes foram divididos entre grupos com pontuações $\leq 2$

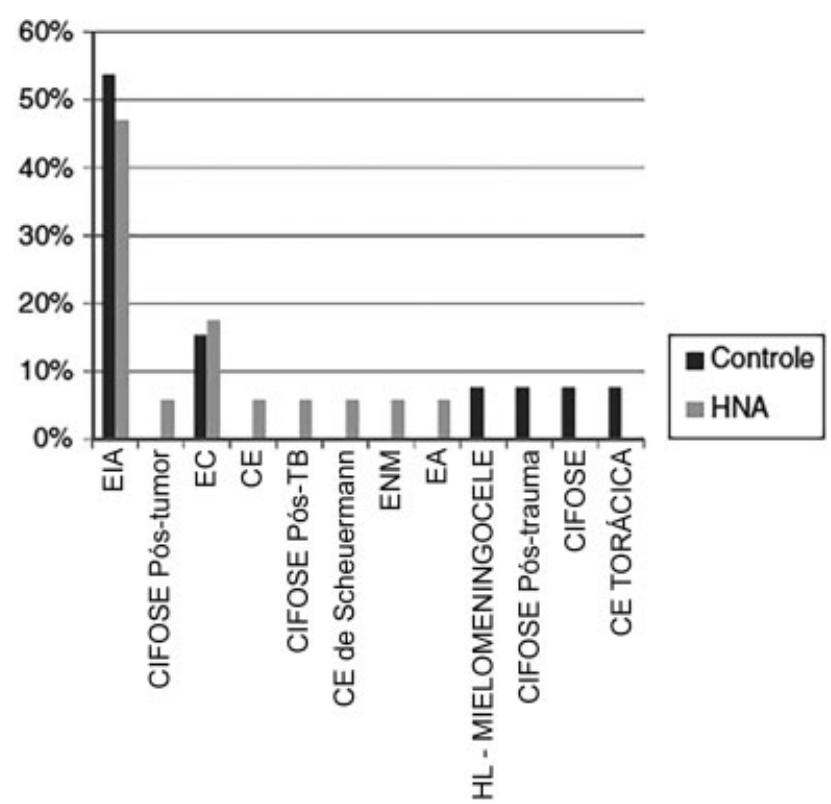

Fig. 2 Etiologia das deformidades em coluna submetidas a cirurgia e sua frequência. Abreviaturas: CE, cifoescoliose; EA, escoliose do adulto; EC, escoliose congênita; EIA, escoliose idiopática do adolescente; ENM, escoliose neuromuscular; HL, hiperlordose; HNA, hemodiluição normovolêmica aguda; TB, tuberculose.

Tabela 1 Sangramento médio por osteotomia, comparação entre os grupos

\begin{tabular}{|l|l|l|l|l|}
\hline & $\mathbf{n}$ & & $\begin{array}{l}\text { Sangramento } \\
\text { médio em } \mathbf{~ m L}\end{array}$ & $\begin{array}{c}\text { Valor } \\
\text { de } \boldsymbol{p}^{\mathbf{a}}\end{array}$ \\
\hline Nenhuma OTT & 5 & HNA & 450 & 0,161 \\
\hline & 5 & Controle & 737,75 & \\
\hline OTT Peso $\leq 2$ & 6 & HNA & 816,6 & 0,412 \\
\hline & 3 & Controle & 633,3 & \\
\hline OTT Peso $>3$ & 6 & HNA & 591 & 0,175 \\
\hline & 5 & Controle & $880^{\text {a }}$ & \\
\hline Total OTT & 12 & HNA & 704,16 & 0,339 \\
\hline & 8 & Controle & 772,2 & \\
\hline
\end{tabular}

Abreviaturas: HNA, hemodiluição normovolêmica aguda; OTT, osteotomia.

Nota: a Uma paciente do grupo foi submetida a três osteotomias com ressecção do corpo vertebral, e apresentou sangramento elevado.

ou $>3$. Na comparação isolada entre os grupos, houve maior média de sangramento no grupo de controle, porém com maior variação, conforme evidenciado na - Figura 3. Houve maior sangramento em ambos os grupos quando feita a osteotomia, sem significância estatística.

Em relação à quantidade de níveis operados, dividiu-se entre grupos com limite maior ou menor do que 10 , e houve mais sangramento no grupo de controle, com mais níveis $(594 \times 920 \mathrm{~mL} ; p=0,095)$, enquanto no caso de menos níveis o sangramento foi menor e similar entre os grupos de HNA e de controle, o que sugere que a HNA seria efetiva para reduzir o sangramento em cirurgias de maior porte. Foi feito o cálculo da densidade do implante (número de parafusos 


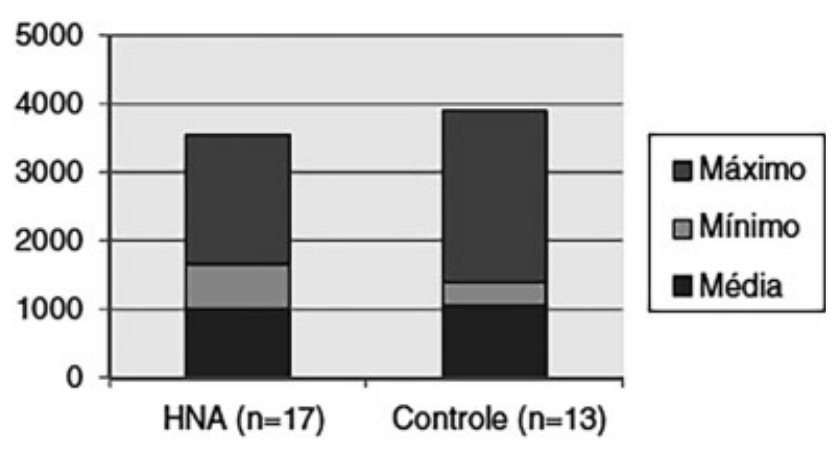

Fig. 3 Sangramento intraoperatório entre os grupos. Teste não paramétrico de Mann-Whitney $(p=0,934)$. Abreviatura: HNA, hemodiluição normovolêmica aguda.

por níveis operados), no qual 1 representa a instrumentação de todos os pedículos operados, e 0 representaria uma cirurgia sem instrumentação (-Tabela 2). Em pacientes do grupo de controle submetidos à osteotomia posterior, houve aumento da necessidade de transfusão $(p>0,05)$. A análise do grupo de HNA mostrou que a maioria não necessitou de transfusão mesmo após ter sido submetida à osteotomia $(p>0,05)$ (-Tabela 3 ). Pacientes transfundidos tiveram maior média de níveis operados, em especial no grupo de controle $(p>0,05)$ ( - Tabela 4$)$.

Para o grupo de HNA, o valor médio de sangramento intraoperatório foi de $629,4 \mathrm{~mL}$, e, no pós-operatório, de $379,11 \mathrm{~mL}$; no grupo de controle, esses valores foram de 754,2 mL e 296,2 mL respectivamente, porém, sem significância estatística. Houve menor redução de sangramento perioperatório-pós-operatório no grupo de HNA, $\operatorname{com} p<0,05$ (- Fig. 4).

Foram comparados os exames laboratoriais antes e após a cirurgia e entre os grupos, que estão representados na - Tabela 5 .
A análise do perfil de coagulação foi feita, de modo que em ambos os grupos houve alargamento do TAP - com maior diferença antes e após a cirurgia no grupo de HNA $(p=0,014)$ - e diminuição do TTPA, com maior queda no grupo de controle $(p=0,793)$ (-Figs. 5 e 6 ).

Houve maior necessidade de uso de hemostáticos locais no grupo de controle em comparação com o HNA (30,8\% versus $5,9 \%$, respectivamente; $p>0,05)$. Não houve relevância estatística para a necessidade de transfusão de acordo com o método aplicado, conforme mostra a - Figura 7. De modo geral, os pacientes que precisaram de hemoderivados apresentavam deformidades mais graves em $75 \%$ dos casos, consequentemente com mais níveis a serem abordados (7-14; média: 11,1), mais osteotomias (1-9; média: 4 versus $0-5$; média: 1,56$)$, com $p<0,05$. Esse grupo também apresentou altas taxas de sangramento perioperatório $(600-2.000 \mathrm{~mL}$; média: $1.050 \mathrm{~mL}$ ), que, quando comparadas com a média de sangramento de pacientes que não receberam transfusão $(593,6 \mathrm{~mL})$, houve significância estatística $(p=0,007)$. Para fins de comparação, os pacientes foram divididos entre grupo com EIA e grupo com demais diagnósticos. Houve maior tempo cirúrgico para o segundo grupo $(p<0,05)$, maior taxa de densidade do implante $(p=0,07)$, e quase o dobro de osteotomias posteriores feitas ( $p>0,05$; - Tabela 6$)$.

\section{Discussão}

O controle do sangramento deve fazer parte do planejamento cirúrgico inicial. A aplicação de técnicas pode prevenir a necessidade de transfusão em pacientes submetidos à cirurgia de EIA. ${ }^{6}$ A evolução de técnicas cirúrgicas possibilitou melhores resultados estéticos e funcionais; porém, quanto mais extenso o procedimento, maior o sangramento perioperatório $^{7-9}$ e a fibrinólise, com consequente aumento da

Tabela 2 Densidade do implante (número de parafusos em relação à quantidade de níveis operados), e comparação entre os grupos

\begin{tabular}{|l|l|l|l|l|l|l|l|l|}
\hline & & $\mathbf{n}$ & Média & Mediana & Mínimo & Máximo & Desvio padrão & Valor de $\boldsymbol{p}^{\mathbf{a}}$ \\
\hline Densidade do implante & HNA & 17 & 0,815 & 0,792 & 0,625 & 1 & 0,141 & 0,146 \\
\hline & Controle & 13 & 0,739 & 0,722 & 0,545 & 1 & 0,131 & \\
\hline
\end{tabular}

Abreviatura: HNA, hemodiluição normovolêmica aguda.

Nota: ${ }^{a} p>0,05$.

Tabela 3 Osteotomia versus necessidade de transfusão, comparação entre os grupos

\begin{tabular}{|l|l|l|l|l|l|l|l|l|}
\hline Transfusão & \multicolumn{2}{|l|}{ Osteotomias - HNA } & \multicolumn{2}{l|}{$\begin{array}{l}\text { Osteotomias - } \\
\text { Controle }\end{array}$} & \multicolumn{2}{l|}{$\begin{array}{l}\text { OTT posterior - } \\
\text { Controle }\end{array}$} & \multicolumn{2}{l|}{ OTT posterior - HNA } \\
\hline & Nenhum & $\begin{array}{l}\text { Pelo menos } \\
\text { um }\end{array}$ & Nenhum & $\begin{array}{l}\text { Pelo menos } \\
\text { um }\end{array}$ & $\begin{array}{l}\text { Nenhum } \\
\text { Pelo menos } \\
\text { um }\end{array}$ & $\begin{array}{l}\text { Nenhum } \\
\text { um }\end{array}$ & $\begin{array}{l}\text { melo mos } \\
\text { um }\end{array}$ \\
\hline Não & $5(100 \%)$ & $10(83,3 \%)$ & $5(100 \%)$ & $4(50 \%)$ & $7(100 \%)$ & $2(33,3 \%)$ & $7(87,5 \%)$ & $8(88,9 \%)$ \\
\hline Sim & 0 & $2(16,7 \%)$ & 0 & $4(50 \%)$ & 0 & $4(66,7 \%)$ & $1(12,5 \%)$ & $1(11,1 \%)$ \\
\hline Total & 5 & 12 & 5 & 8 & 7 & 6 & 8 & 9 \\
\hline Valor de $p$ & 1 & & 0,105 & & $0,021^{\text {a }}$ & & 1 & \\
\hline
\end{tabular}

Abreviaturas: HNA, hemodiluição normovolêmica aguda; OTT, oosteotomia.

Nota: ${ }^{a}$ Valor de $p$ calculado pelo teste exato de Fisher $(p<0,05)$. 
Tabela 4 Número de níveis operados versus necessidade de transfusão, comparação entre os grupos

\begin{tabular}{|c|c|c|c|c|c|c|c|c|}
\hline & \multirow[t]{2}{*}{ Transfusão } & \multicolumn{6}{|c|}{ Níveis operados } & \\
\hline & & $\mathbf{n}$ & Média & Mediana & Mínimo & Máximo & Desvio padrão & \\
\hline \multirow[t]{2}{*}{ HNA } & Não & 15 & 10,7 & 11 & 7 & 16 & 2,7 & $p>0,05$ \\
\hline & Sim & 2 & 10,5 & 10,5 & 7 & 14 & 4,9 & \\
\hline \multirow[t]{2}{*}{ Controle } & Não & 9 & 8,9 & 9 & 4 & 11 & 2,1 & $p=0.020$ \\
\hline & Sim & 4 & 11,5 & 11 & 11 & 133 & 1,0 & \\
\hline
\end{tabular}

Abreviatura: HNA, hemodiluição normovolêmica aguda.

Nota: Teste não paramétrico de Mann-Whitney $(p<0,05)$.

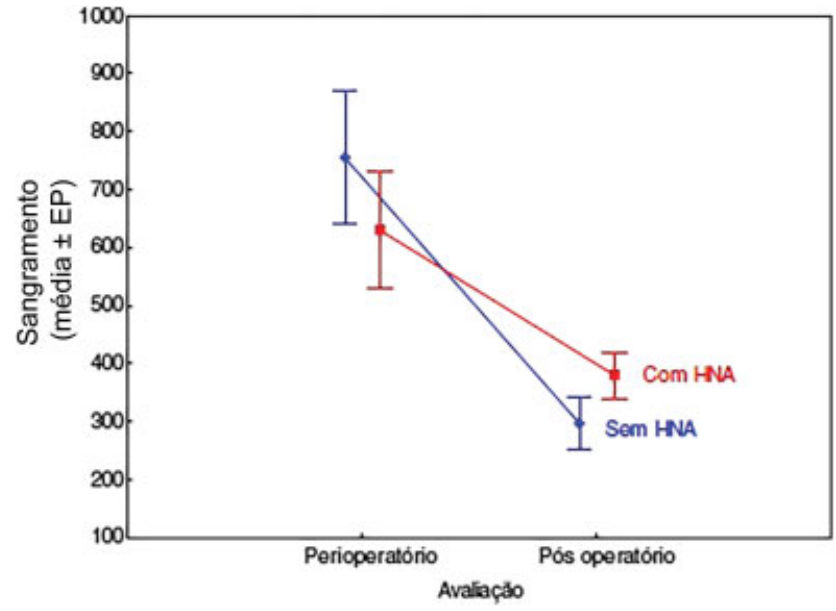

Fig. 4 Avaliação do sangramento perioperatório versus pós-operatório na comparação entre os grupos. Abreviaturas: EP, erro padrão; HNA, hemodiluição normovolêmica aguda.

hemorragia, o que gera um círculo vicioso que eleva a morbimortalidade. ${ }^{9}$

Na cirurgia de escoliose neuromuscular (ENM), há maior perda sanguínea em comparação com a de EIA, ${ }^{10-12}$ principalmente pela maior extensão da artrodese, ${ }^{11}$ com possível relação com uso de anticonvulsivantes e subnutrição. A perda sanguínea estimada na cirurgia para EIA é de $1.300 \mathrm{~mL}$ a $2.200 \mathrm{~mL}$, e, na ENM, é de $2.000 \mathrm{~mL}$ a $4.000 \mathrm{~mL} .^{12}$ A perda sanguínea, além da hipotensão, anemia e coagulopatia por depleção, leva a aumento da quantidade de unidades transfundidas. ${ }^{10}$ No presente estudo, os pacientes com EIA tiveram média de sangramento de $561 \mathrm{~mL}$, enquanto em outras deformidades o sangramento chegou a $2.000 \mathrm{~mL}$ (média: $806 \mathrm{~mL}$ ). Somente um paciente com EIA necessitou de transfusão.

Espera-se prolongamento de TAP e TTPA pós-operatórios pela disfunção da agregação plaquetária, que, principalmente na ENM, ${ }^{10,13}$ indica sobrerregulação da coagulação em resposta ao estresse cirúrgico e ao consumo de fatores de coagulação. ${ }^{13}$ No presente estudo, houve diminuição do TTPA e prolongamento doTAPem todos os pacientes, sem repercussões clínicas, como constatado por Oppitz e Stefani. ${ }^{5}$

O sangramento nas correções de deformidade é elevado devido à rica vascularização local, ampla exposição, e ao tempo cirúrgico prolongado. A perda sanguínea estimada é de $10-30 \mathrm{~mL} / \mathrm{kg} .{ }^{11,14}$ Houve muita variabilidade nos valores encontrados neste estudo (200-2.000 mL), fato que pode ser atribuído à etiologia, à gravidade da deformidade, aos níveis operados, às osteotomias, e ao tempo cirúrgico. Sexo, idade avançada, doença cardiovascular, laminectomias extensas e Ht baixo no pré-operatório ${ }^{15,16}$ não resultaram em aumento do sangramento.

As osteotomias são eventualmente necessárias para corrigir deformidades mais rígidas, especialmente em adultos. ${ }^{17} \mathrm{~A}$ subtração pedicular está associada a maiores perdas sanguíneas (até $3 \mathrm{Ls}$ ) pela dissecção de veias epidurais calibrosas ${ }^{18} \mathrm{e}$ tempo cirúrgico aumentado. ${ }^{8}$ A RCV é uma opção para deformidades graves e/ou rígidas. ${ }^{17}$ Osteotomias posteriores (também conhecidas como osteotomias de Smith Petersen), consideradas mais fáceis e rápidas, têm poder de correção limitado (5o-20。), enquanto a de subtração pedicular pode corrigir 30॰-40。 por nível. ${ }^{7,19}$ Há relatos de maior taxa de sangramento em abordagem posterior do que em anterior. ${ }^{12}$ Todos os pacientes do presente estudo foram abordados por via posterior.

Neste estudo, os pacientes submetidos a osteotomias tiveram uma média de sangramento superior, como na literatura, mas o único dado estatisticamente relevante foi o aumento da necessidade de transfusão no grupo de controle quando feitas osteotomias posteriores. A HNA não se provou eficaz para evitar a transfusão quando feita a osteotomia, porém notou-se tendência à relevância estatística quanto mais agressiva foi a abordagem.

Identificar fatores de risco, descontinuar medicações como ácido acetilsalicílico, anti-inflamatórios e anticoagulantes, ${ }^{1,20}$ e até embolização pré-operatória de corpo vertebral ${ }^{21}$ diminuem o tempo de internamento, os custos, e o sangramento. A dissecção minuciosa do periósteo e o uso de eletrocautério e de agentes hemostáticos ${ }^{1,6}$ podem ser adjuvantes da doação autóloga, HNA ou anestesia hipotensiva. ${ }^{6}$ Embora Szpalski et $\mathrm{al}^{22}$ e Urban et $\mathrm{al}^{23}$ tenham mostrado que existem evidências adequadas para seu uso, a hipotensão controlada é controversa em cirurgias da coluna, por não diminuir a perda sanguínea intraoperatória ${ }^{14}$ e pelo risco de lesão medular por redução do fluxo. ${ }^{7,24}$ Os autores mencionam também que o uso de agentes hemostáticos sistêmicos e locais seria controverso. $^{22}$

O uso de fibrinolíticos ganhou popularidade nos anos 1990. ${ }^{18,25}$ Em crianças com ENM, seu uso foi eficaz na diminuição de sangramento e transfusão. ${ }^{10,26}$ A aprotinina inibe enzimas anticoagulantes, e inibe a via intrínseca da coagulação e a agregação plaquetária. ${ }^{9,25}$ Seu uso foi descontinuado ${ }^{9,10}$ 
Hemodiluição Normovolêmica Aguda em Cirurgias Batista et al. 521

Tabela 5 Média de valores comparados entre os grupos em diferentes momentos de avaliação

\begin{tabular}{|c|c|c|c|c|c|c|c|}
\hline & $\begin{array}{l}\text { Momento da } \\
\text { avaliação }\end{array}$ & $\begin{array}{l}\text { HNA sem } \\
\text { transfusão }\end{array}$ & $\begin{array}{l}\text { HNA com } \\
\text { transfusão }\end{array}$ & $\begin{array}{l}\text { Controle sem } \\
\text { transfusão }\end{array}$ & $\begin{array}{l}\text { Controle com } \\
\text { transfusão }\end{array}$ & $\begin{array}{l}\text { Total sem } \\
\text { transfusão }\end{array}$ & $\begin{array}{l}\text { Total com } \\
\text { transfusão }\end{array}$ \\
\hline$n$ & & 15 & 2 & 9 & 4 & 30 & 30 \\
\hline \multirow[t]{5}{*}{$\mathrm{HB}$} & Pré-operatório & 13,7 & 14,4 & 13,5 & 12,5 & 13,6 & 13,1 \\
\hline & Pós-operatório & 10,8 & 7,8 & 11,9 & 10,4 & 11,1 & 9,5 \\
\hline & Após $24 \mathrm{~h}$ & 9,6 & 6,0 & 10,3 & 10,4 & 9,8 & 8,9 \\
\hline & Pós-pré & $-2,9$ & $-6,7$ & $-1,8$ & $-2,1$ & $-2,6$ & $-3,6$ \\
\hline & Após 24 h-pré & $-4,1$ & $-8,5$ & $-3,4$ & $-2,1$ & $-3,9$ & $-4,2$ \\
\hline \multirow[t]{5}{*}{ HT } & Pré-operatório & 40,8 & 43,3 & 40,4 & 37,7 & 40,6 & 39,6 \\
\hline & Pós-operatório & 32,2 & 23,0 & 35,7 & 32,7 & 33,3 & 29,4 \\
\hline & Após $24 \mathrm{~h}$ & 28,6 & 18,0 & 30,7 & 30,8 & 29,3 & 26,5 \\
\hline & Pós-pré & $-8,6$ & $-20,3$ & $-5,0$ & $-5,0$ & $-7,5$ & $-10,1$ \\
\hline & Após 24 h-pré & $-12,2$ & $-25,3$ & $-10,3$ & $-6,9$ & $-11,5$ & $-13,0$ \\
\hline \multirow[t]{3}{*}{ TAP } & Pré-operatório & 12,5 & 11,9 & 14,0 & 12,4 & 13,0 & 12,2 \\
\hline & Após $24 \mathrm{~h}$ & 14,5 & 14,0 & 14,9 & 14,6 & 14,6 & 14,4 \\
\hline & Após 24 h-pré & 2,0 & 2,1 & 1,0 & 2,3 & 1,7 & 2,2 \\
\hline \multirow[t]{3}{*}{ TTPA } & Pré-operatório & 32,7 & 31,3 & 29,7 & 25,3 & 31,6 & 27,3 \\
\hline & Após $24 \mathrm{~h}$ & 30,9 & 32,9 & 26,9 & 24,7 & 29,6 & 27,4 \\
\hline & Após 24 h-pré & $-1,7$ & 1,6 & $-2,5$ & $-0,6$ & $-2,0$ & 0,1 \\
\hline
\end{tabular}

Abbreviaturas: HB, hemoglobina sérica em g/dL; HNA, hemodiluição normovolêmica aguda; HT, hematócrito em \%; TAP, tempo de atividade da protrombina, em segundos; TTPA, tempo de tromboplastina parcial ativada, em segundos.

Nota: Em todos os grupos, $p>0,05$.

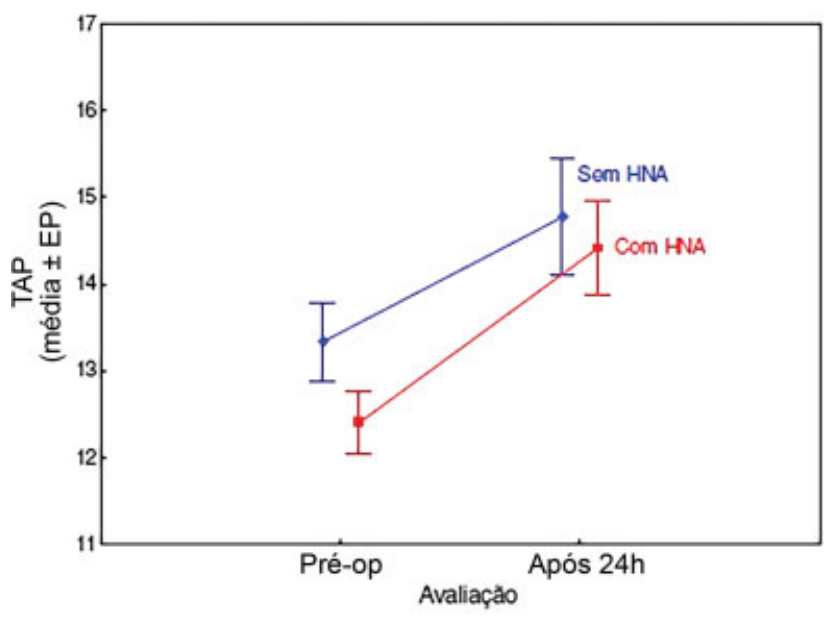

Fig. 5 Prolongamento dos valores do tempo de atividade da protrombina em ambos os grupos no período pós-operatório de $24 \mathrm{~h}(p>0,05)$. Abreviaturas: EP, erro padrão; HNA, hemodiluição normovolêmica aguda; pré-op, pré-operatório; TAP, tempo de atividade da protrombina.

devido ao aumento da mortalidade por infarto agudo do miocárdio ${ }^{9}$ e insuficiência renal aguda, ${ }^{25}$ apesar de estudos prévios demonstrarem a redução da transfusão em cirurgias cardíacas, de joelho e de quadril, ${ }^{9,18,25}$ e também apesar de ela ter sido considerada superior ao ácido tranexâmico em osteotomias pediculares. ${ }^{18}$

O ácido tranexâmico foi administrado em todos os pacientes deste estudo. Ele age pela ligação reversível do plasminogênio com a lisina, ${ }^{24}$ e é considerado seguro, muito usado em cirurgias

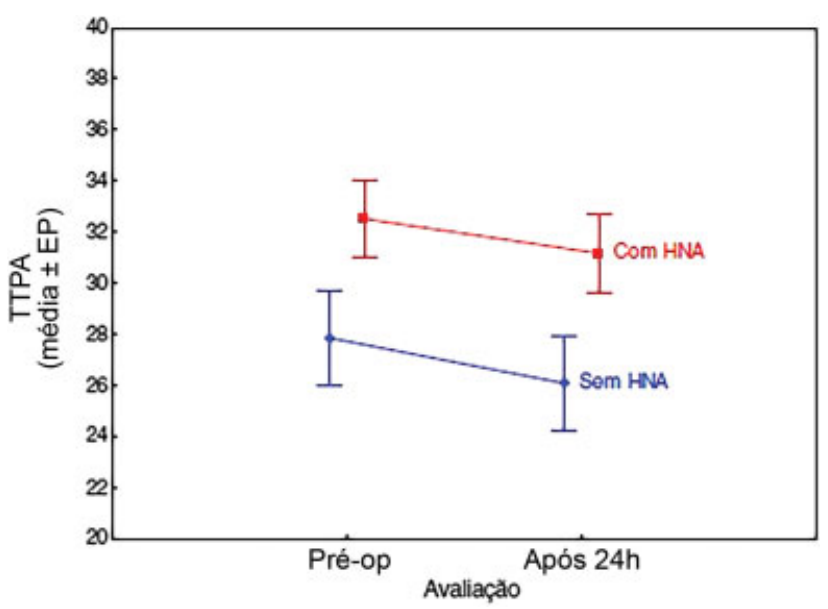

Fig. 6 Queda dos valores de tempo de tromboplastina parcial ativada em ambos os grupos após $24 \mathrm{~h}$ da cirurgia $(p>0,05)$. Abreviaturas: EP, erro padrão; HNA, hemodiluição normovolêmica aguda; pré-op, préoperatório; TTPA, tempo de tromboplastina parcial ativada.

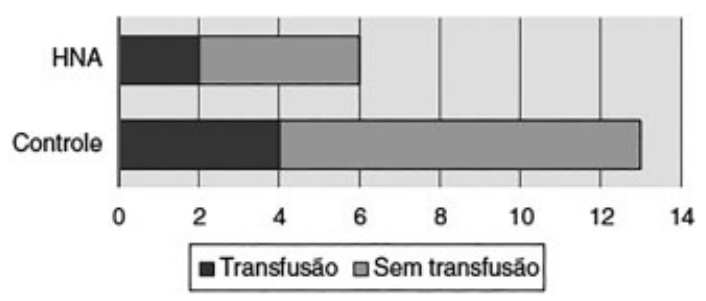

Fig. 7 Necessidade de transfusão em comparação com a hemodiluição $(p=0,360)$. Abreviatura: HNA, hemodiluição normovolêmica aguda. 
Tabela 6 Comparação entre parâmetros relevantes de pacientes com diagnóstico de escoliose idiopática do adolescente versus demais deformidades

\begin{tabular}{|l|l|l|l|}
\hline & $\begin{array}{l}\text { Escoliose } \\
\text { idiopática } \\
\text { do adolescente } \\
(\mathbf{n}=\mathbf{1 4})\end{array}$ & $\begin{array}{l}\text { Outras } \\
\text { deformidades } \\
(\mathbf{n}=\mathbf{1 6})\end{array}$ & $p$-value \\
\hline $\begin{array}{l}\text { Tempo } \\
\text { cirúrgico } \\
\text { (média) }\end{array}$ & $4,29 \mathrm{~h}$ & $5,45 \mathrm{~h}$ & 0,002 \\
\hline $\begin{array}{l}\text { Densidade do } \\
\text { implante } \\
\text { (média) }\end{array}$ & 0,74 & 0,82 & 0,07 \\
\hline $\begin{array}{l}\text { Osteotomias } \\
\text { (média) }\end{array}$ & 0,92 & 1,86 & $>0,05$ \\
\hline $\begin{array}{l}\text { Sangramento } \\
\text { (média) }\end{array}$ & $561 \mathrm{ml}$ & $806 \mathrm{ml}$ & 0,058 \\
\hline
\end{tabular}

cardíacas e uroginecológicas. Em estudo retrospectivo, ${ }^{24}$ seu uso na EIA resultou em menos sangramento e transfusão; ${ }^{24}$ porém, não se mostrou efetivo na subtração pedicular em adultos. ${ }^{18}$ Seu uso não aumenta a morbimortalidade e a incidência de eventos tromboembólicos. ${ }^{27}$

No presente estudo, taxa de transfusão sanguínea homóloga encontrada foi de $20 \%$, que se encontra dentro da margem de $8 \%-36 \%$ relatada por Purvis et al, ${ }^{28}$ que demonstraram múltiplas complicações possíveis (aumento de mortalidade, infecções hospitalares, prolongamento do internamento, além do custo elevado), 7,16,20,29 apesar de nenhuma complicação ter sido relatada. Não foi possível isolar um fator responsável pela transfusão, mas uma associação variável entre níveis operados, gravidade da deformidade, tempo cirúrgico, e osteotomias.

A transfusão autóloga é considerada mais segura, ${ }^{3}$ a ser feita com doação prévia à cirurgia ( 3 a 5 semanas, com limitação em idosos e anêmicos), ${ }^{7}$ hemodiluição normovolêmica, preservação celular (custo elevado) 7,16,28 ou hemodiluição hipervolêmica, que reduz a necessidade de transfusão alogênica ${ }^{14}$ por meio da diluição em plasma ou em soluções de macromoléculas, com aumento do volume circulante. ${ }^{4}$ É considerada rápida, mais fácil, mais estável, e mais barata do que a HNA.

Na HNA, o sangue coletado é diluído com fluido acelular na proporção de $2-4: 1,7,16$ e ela leva à redução da perda sanguínea perioperatória com manutenção do fluxo. ${ }^{3-5}$ Muitos estudos mostram que a HNA reduz a necessidade de transfusão homóloga entre $18 \%$ e $90 \% ;^{5,29}$ porém, no presente estudo, não houve significância estatística quanto a isso, talvez pelo número de casos. A HNA é considerada segura e eficaz nas cirurgias de coluna quando há estimativa de perda maior do que $1 \mathrm{~L}$ ou $20 \%$ do volume sanguíneo. ${ }^{16,29}$ Há o risco de hemodiluição extrema ( $\mathrm{Ht}<20 \%$ ), com risco de isquemia tecidual, revertida com infusão de plasma fresco, segundo McLaughlin. ${ }^{14}$ Seu uso na população pediátrica foi testado em estudo $^{30}$ prospectivo randomizado de 2004 com crianças submetidas à artrodese por via posterior, que provou que a HNA é segura e capaz de reduzir a necessidade de transfusão, sem complicações relacionadas à anemia. ${ }^{30}$ Tse et $a^{31}$ mos- traram em sua revisão que HNA, ácido tranexâmico, morfina intratecal e modificação das técnicas operatórias parecem ser as melhores opções para reduzir sangramento perioperatório e transfusão sanguínea alogênica. O uso da HNA no presente estudo auxiliou no controle do sangramento, porém não a ponto de evitar a transfusão isoladamente.

\section{Conclusão}

A técnica de HNA associada ao ácido tranexâmico não se provou eficaz em reduzir a necessidade de transfusão sanguínea homóloga em cirurgias corretivas de deformidades da coluna, apesar da tendência de reduzir o sangramento intraoperatório, principalmente nos casos considerados mais complexos. 0 efeito combinado da gravidade da deformidade, das osteotomias e do número de níveis operados/instrumentados é fator determinante da necessidade de transfusão, e a associação de medidas pré e intraoperatórias para controle de sangramento deve ser considerada nesses casos. Acredita-se que uma casuística maior poderia comprovar sua eficácia na comparação com fibrinolíticos isolados.

\section{Conflitos de Interesse}

Os autores declaram não haver conflitos de interesse.

\section{Referências}

1 Baird EO, McAnany SJ, Lu Y, Overley SC, Qureshi SA. Hemostatic agents in spine surgery: A critical analysis review. JBJS Rev 2015;3(01):X

2 Maxwell MJ, Wilson MJA. Complications of blood transfusion. BJA Educ 2006;6(06):225-229

3 Walunj A, Babb A, Sharpe R. Autologous blood transfusion. BJA Educ 2006;6(05):192-196

4 Oriani G, Pavesi M, Oriani A, Bollina I. Acute normovolemic hemodilution. Transfus Apheresis Sci 2011;45(03):269-274

5 Oppitz PP, Stefani MA. Acute normovolemic hemodilution is safe in neurosurgery. World Neurosurg 2013;79(5-6):719-724

6 Verma RR, Williamson JB, Dashti H, Patel D, Oxborrow NJ. Homologous blood transfusion is not required in surgery for adolescent idiopathic scoliosis. JBone Joint Surg $\mathrm{Br}$ 2006;88 (09):1187-1191

7 Kim KT, Park KJ, Lee JH. Osteotomy of the spine to correct the spinal deformity. Asian Spine J 2009;3(02):113-123

8 Liu H, Yang C, Zheng Z, et al. Comparison of Smith-Petersen osteotomy and pedicle subtraction osteotomy for the correction of thoracolumbar kyphotic deformity in ankylosing spondylitis: a systematic review and meta-analysis. Spine 2015;40(08):570-579

9 Khurana A, Guha A, Saxena N, Pugh S, Ahuja S. Comparison of aprotinin and tranexamic acid in adult scoliosis correction surgery. Eur Spine J 2012;21(06):1121-1126

10 Dhawale AA, Shah SA, Sponseller PD, et al. Are antifibrinolytics helpful in decreasing blood loss and transfusions during spinal fusion surgery in children with cerebral palsy scoliosis? Spine 2012;37(09):E549-E555

11 Modi HN, Suh S-W, Hong J-Y, Song S-H, Yang J-H. Intraoperative blood loss during different stages of scoliosis surgery: A prospective study. Scoliosis 2010;5(01):16-16

12 Shapiro F, Sethna N. Blood loss in pediatric spine surgery. Eur Spine J 2004;13(Suppl 1):S6-S17

13 Bosch P, Kenkre TS, Londino JA, Cassara A, Yang C, Waters JH. Coagulation Profile of Patients with Adolescent Idiopathic Scoliosis Undergoing Posterior Spinal Fusion. J Bone Joint Surg Am 2016;98 (20):e88 
14 El-Dessouky MI, Waly SH, Nasr YM. Acute normovolemic hemodilution in spinal fusion surgery. Egypt J Anaesth 2011;31:249-254

15 Epstein NE. Bloodless spinal surgery: a review of the normovolemic hemodilution technique. Surg Neurol 2008;70(06): 614-618

16 Stehling L, Zauder HL. Acute normovolemic hemodilution. Transfusion 1991;31(09):857-868

17 Enercan M, Ozturk C, Kahraman S, Sarıer M, Hamzaoglu A, Alanay A. Osteotomies/spinal column resections in adult deformity. Eur Spine J 2013;22(Suppl 2):S254-S264

18 Baldus CR, Bridwell KH, Lenke LG, Okubadejo GO. Can we safely reduce blood loss during lumbar pedicle subtraction osteotomy procedures using tranexamic acid or aprotinin? A comparative study with controls. Spine 2010;35(02):235-239

19 Hyun S-J, Rhim S-C. Clinical outcomes and complications after pedicle subtraction osteotomy for fixed sagittal imbalance patients : a long-term follow-up data. J Korean Neurosurg Soc 2010;47(02):95-101

20 Blanchette CM, Wang PF, Joshi AV, Asmussen M, Saunders W, Kruse P. Cost and utilization of blood transfusion associated with spinal surgeries in the United States. Eur Spine J 2007;16(03): 353-363

21 Tuchman A, Mehta VA, Mack WJ, Acosta FL Jr. Novel application of pre-operative vertebral body embolization to reduce intraoperative blood loss during a three-column spinal osteotomy for non-oncologic spinal deformity. JClin Neurosci 2015;22(04):765-767

22 Szpalski M, Gunzburg R, Sztern B. An overview of blood-sparing techniques used in spine surgery during the perioperative period. Eur Spine J 2004;13(Suppl 1):S18-S27
23 Urban MK, Beckman J, Gordon M, Urquhart B, Boachie-Adjei O. The efficacy of antifibrinolytics in the reduction of blood loss during complex adult reconstructive spine surgery. Spine 2001; 26(10):1152-1156

24 Yagi M, Hasegawa J, Nagoshi N, et al. Does the intraoperative tranexamic acid decrease operative blood loss during posterior spinal fusion for treatment of adolescent idiopathic scoliosis? Spine 2012;37(21):E1336-E1342

25 Tayyab NA, Marillier MM, Rivlin M, et al. Efficacy of aprotinin as a blood conservation technique for adult deformity spinal surgery: a retrospective study. Spine 2008;33(16):1775-1781

26 Cole JW, Murray DJ, Snider RJ, Bassett GS, Bridwell KH, Lenke LG. Aprotinin reduces blood loss during spinal surgery in children. Spine 2003;28(21):2482-2485

27 da Rocha VM, de Barros AGC, Naves CD, et al. Use of tranexamic acid for controlling bleeding in thoracolumbar scoliosis surgery with posterior instrumentation. Rev Bras Ortop 2015;50(02):226-231

28 Purvis TE, Goodwin CR, De la Garza-Ramos R, et al. Effect of liberal blood transfusion on clinical outcomes and cost in spine surgery patients. Spine J 2017;17(09):1255-1263

29 Fischer ST, Schuroff AA, Vialle LR. Hemodiluição isovolumétrica no tratamento cirúrgico da escoliose idiopática. Rev Bras Ortop 1994;29(03):107-110

30 Oliveira GS, Tenório SB, Cumino DO, et al. Acute normovolemic hemodilution in children submitted to posterior spinal fusion. Rev Bras Anestesiol 2004;54(01):84-90

31 Tse EY, Cheung WY, Ng KF, Luk KD. Reducing perioperative blood loss and allogeneic blood transfusion in patients undergoing major spine surgery. J Bone Joint Surg Am 2011;93(13):1268-1277 